\title{
Vers une nouvelle autobiographie: subversions et transformations du genre dans les autobiographies contemporaines françaises
}

\author{
Towards a new autobiography: subversions \\ and transformations of the genre in French \\ contemporary autobiographies
}

\author{
ANTONELLA LiPSCOMB \\ $\mathrm{Ph}$.D. University of Oxford \\ antonella_lipscomb@hotmail.com
}

\begin{abstract}
Autobiography is a genre that Philippe Lejeune defines as the retrospective record in prose that a real person gives to his or her own being, emphasizing the personal life and in particular the story of life (Lejeune, 1975: 14). However, what characterizes the French autobiographies of the past 50 years studied in this article is their desire to detach themselves, even deny the genre adopted for all the negative connotations related to the autobiographical genre. Accused of individualism, egocentrism and narcissism, autobiography is a difficult genre to assume and its values of authenticity and sincerity difficult to guarantee. Attraction and aversion are the contradictory feelings experienced by the autobiographers examined in this article in relation to the genre adopted. Feelings that manifest themselves through the resistance to adhere to any definition, the desire to blur the boundaries between autobiography, biography, self-portrait, journal and fiction, and through a fascinating use of personal pronouns in the narration.
\end{abstract}

\begin{abstract}
Résumé
L'autobiographie est ce genre qui d'après Philippe Lejeune se définit comme un "récit rétrospectif en prose qu'une personne réelle fait de sa propre existence lorsqu'elle met l'accent sur sa vie individuelle, en particulier sur l'histoire de sa propre personnalité" (Lejeune, 1975: 14). Ce qui caractérise les autobiographies françaises des 50 dernières années étudiées dans cet article est leur désir de se détacher, voire nier le genre adopté pour toutes les connotations négatives auxquelles ce genre est attaché: accusée d'individualisme, égocentrisme, narcissisme, l'autobiographie est un genre difficile à assumer et ses valeurs d'authenticité et de sincérité difficiles à assurer. Attraction et répulsion sont les sentiments contradictoires éprouvés par les autobiographes examinés dans cet article par rapport au genre adopté ; des sentiments qui se manifestent par la résistance à toute définition, le désir de brouiller les frontières entre autobiographie, biographie, autoportrait, journal et fiction, et par un jeu fascinant de pronoms personnels dans la narration.
\end{abstract}


Anales de Filología Francesa, n. ${ }^{\circ}$ 27, 2019

VERS UNE NOUVELLE AUTOBIOGRAPHIE: SUBVERSIONS ET TRANSFORMATIONS DU GENRE DANS...

Key-words

Autobiography, French contemporary literature.

\section{Mots-clés}

Autobiographie, littérature contemporaine française.

\section{Introduction}

Dans Le Pacte autobiographique Philippe Lejeune définit l'autobiographie comme un récit rétrospectif en prose qu'une personne réelle fait de sa propre existence lorsqu'elle met l'accent sur sa vie individuelle, en particulier sur l'histoire de sa propre personnalité' (Lejeune, 1975: 14). Le pacte autobiographique, précise Lejeune, c'est l'affirmation dans le texte de "l'identité de l'auteur, du narrateur et du personnage" (Lejeune, 1975: 26). Or ce qui caractérise les autobiographies étudiées dans cet article est leur désir de se détacher, voire nier le genre adopté pour toutes les connotations négatives auxquelles l'autobiographie est attachée. Accusée d'individualisme, égocentrisme, narcissisme, l'autobiographie est un genre difficile à assumer et ses valeurs d'authenticité et de sincérité difficiles à assurer. "C $C$ 'est dangereux, l'autobiographie", affirme Patrick Modiano, “c'est un genre bâtard, une solution de facilité pour quand on manque de courage [...] c'est toujours une baisse de tension". Considérée d'autre part comme "le degré le plus bas de la teneur littéraire", l'autobiographie se voit confrontée, d'après Jacques Lecarme, à une véritable idéologie "anti-autobiographique" (Lecarme \& Lecarme-Tabone, 1997: 9). Mais comment résister au désir de se raconter et au plaisir de redécouvrir son passé ? Si d'un côté les autobiographes affirment récuser, comme Alain Robbe-Grillet, "l'entreprise autobiographique, où l'on prétend rassembler toute une existence vécue [...] en un volume clos, sans manques et sans bavures", ils avouent en même temps se sentir attirés et incapables "d'échapper à sa fascination" (Robbe-Grillet, 1984: 58). Attraction et répulsion sont les sentiments contradictoires éprouvés par les autobiographes contemporains français examinés dans cet article par rapport au genre adopté. Une ambigüité qui rapprocherait ces récits à l'autofiction; mais là encore, il s'agirait de se conformer à un genre, se plier aux règles du jeu/je autofictionnel. Ce qui distingue les récits étudiés ici est bien leur résistance à toute définition, leur désir de brouiller les frontières entre autobiographie, biographie, autoportrait journal et fiction, et un jeu fascinant de pronoms personnels dans la narration.

\section{Contre le mot "autobiographie"}

A partir de la seconde moitié du XX siècle, la tendance adoptée par la plupart des autobiographes français consiste à éluder le genre en question par la résistance à toute classification. Lorsqu'en 1963 Julien Green publie son premier volume autobiographique Partir

1 Entretien avec Madeleine Chapsal. 1985. Lire, 12, cité par Jacques Lecarme et Eliane Lecarme-Tabone (1997: 284). 
avant le jour, il s'empresse de préciser dès les premières pages que son "livre" n'est pas "autobiographie pure et simple", mais bien "autre chose":

\begin{abstract}
Si je n'y mettais sans cesse bon ordre, ce livre tomberait dans l'autobiographie pure et simple. Or, c'est bien autre chose que je désire. Je me propose de regarder là où je n'ai jamais tourné les yeux que par hasard, je veux tâcher de voir clair dans cette partie de la conscience qui demeure si souvent obscure à mesure que nous nous éloignons de notre enfance (Green, 1963: 97-98).
\end{abstract}

L'année de sa publication, remarque justement Lecarme, “Green ne pense rien de bon du terme 'autobiographie' et tient à ne pas 'tomber' dans ce qu'il désigne et qui ne peut être que bas" (Lecarme \& Lecarme-Tabone, 1997: 14). Or, lorsqu'en 1984 sont réunis en deux tomes les quatre volumes de son enfance sous le titre Jeunes années. Autobiographie I et II, le terme, constate encore Lecarme, est "assumé" et les connotations négatives "atténuées". Même virement chez Michel Leiris, qui passe dans son premier récit autobiographique L'Age d'homme d'un créateur de "romans autobiographiques" ou "faiseurs de confessions et de Mémoires" redouté (Leiris, 1939: 10 et 51), à un "auteur d'honnêtes essais autobiographiques qui feront peut-être figure de défense et illustration de ce genre littéraire" dans son dernier récit autobiographique Fibrilles (Leiris, 1966: 255). Mais si le mot "autobiographie" est, comme pour Julien Green, finalement assumé c'est, précise Lecarme, plutôt comme un "pis-aller, après avoir renoncé aux deux grandes vocations de sa jeunesse, la poésie et la révolution" (Lecarme \& Lecarme-Tabone, 1997: 17).

Le passage à la première personne du singulier opéré par Julien Green et Michel Leiris est loin d'être une démarche isolée. Nombreux auteurs se tournent vers l'écriture de soi dans les années 1950 et 1960: Simone de Beauvoir dans Mémoires d'une jeune fille rangée (1958), Jean-Paul Sartre dans Les Mots (1964), Violette Leduc dans La Bâtarde (1964) offrent au lecteur une écriture encore caractérisée par le pacte de vérité suggérée par Lejeune. Dans les années 1970 l'autobiographie se place au cœur de la vie littéraire mais commence aussi l'ère du soupçon autobiographique et la remise en question du genre, des frontières entre autobiographie et fiction et conséquemment du pacte de vérité. C'est le cas de Georges Perec, qui alterne chapitres de fiction et autobiographie dans Wou le Souvenir d'enfance (1975); Roland Barthes, qui mêle dans son Roland Barthes par Roland Barthes (1975) biographie, autoportrait et fiction, pour éviter ainsi tout risque d'être classifié comme une autobiographie; et Serge Doubrovsky, qui crée en 1977 le néologisme "autofiction" pour désigner son "roman"Fils (1977), un terme qui repose cette fois-ci sur un pacte "oxymoronique" (Jaccomard, 1993) et qui consiste en une “fiction, d'évènements et de faits strictement réels. Si l'on veut, autofiction, d'avoir confié le langage d'une aventure à l'aventure d'un langage en liberté" (Doubrovsky, 1977).

Au cours des années 80, Alain Robbe-Grillet et Nathalie Sarraute se tournent aussi vers le genre autobiographique, suscitant aussi bien l'étonnement du public que celui de la critique. Revirement spectaculaire, en effet, pour ces initiateurs de la révolution roma- 
nesque des années 1950 qui, comme l'explique Armel, "sortent de leur réputation de purs théoriciens de l'écriture, soi-disant irréductibles à tout discours sur eux-mêmes, en livrant au public des livres à la première personne, qui ne s'intitulent plus ni 'roman' ni 'récit', ni encore moins 'essai', où le narrateur-personnage principal est clairement identifié à l'auteur" (Armel, 1990b: 25). Au sujet de Enfance (1983), Nathalie Sarraute se défend: “Il ne s'agit pas d'une autobiographie. Ce n'est pas un rapport sur toute ma vie” (Forrester, 1983: 19). Ailleurs elle affirme également:

Je n'aime pas l'autobiographie, je n'ai aucune confiance dans les autobiographies, parce qu'on s'y décrit toujours sous un jour [...], on veut se montrer sous un certain jour. Et puis c'est toujours très partial - enfin, moi, je n'y crois jamais ${ }^{2}$.

Mais plus loin, Sarraute rectifie: 'Ecoutez, je crois qu'un des textes que j'admire le plus de tous les textes littéraires, ce sont Les Confessions de Rousseau. Alors, ça suffirait déjà pour que je ne puisse pas dire du mal d'une autobiographie"3. Difficile cependant de ne pas ressentir le gêne qui entoure l'expression "évoquer tes souvenirs d'enfance” que prononce une des voix qui compose Enfance; elle est si sujette au cliché que sa seule prononciation conduit l'auteur à “tortiller" (Sarraute, 1983: 7). Or, c'est bien cette formule que Sarraute choisit pour définir son entreprise: “Oui, je n’y peux rien, ça me tente, je ne sais pas pourquoi [...]” (Sarraute, 1983: 7). Ailleurs, l'auteur explique: “J'ai sélectionné, comme pour tous mes autres livres, des instants dont je pourrais retrouver la sensation. Cette fois, j'ai dit qu'il s'agissait de moi, non pas d'il ou elle" (in Forrester, 1983: 19). Une déclaration qui permet à Sarraute de justifier son recours au "je” en situant son entreprise dans la continuité des œuvres qui l'ont précédée, à savoir la recherche de ces "mouvements" intérieurs qu'elle nomme les "tropismes". Si Sarraute place par conséquent l'expression "souvenirs d'enfance" entre guillemets c'est qu'elle craint que son récit sacrifie ce "mouvement" qui est encore "tout vacillant", "des petits bouts de quelque chose d'encore vivant", pour quelque chose de “fixé une fois pour toutes, du 'tout cuit', donné d'avance” (Sarraute, 1983: 9):

Je n'ai choisi dans Enfance que des souvenirs dans lesquels existaient ces mouvements, mais lorsque j'ai voulu faire revivre mes vacances en Isère, les images se sont animées [...]. Je n'écrivais pas une autobiographie dans laquelle je devais raconter tout ce qui m'était arrivéét.

Aussi le récit se termine-t-il lorsque survient cet "énorme espace très encombré, bien éclairé" de l'adolescence et de la vie adulte, et se construit dans l'effort de saisir une subs-

2 Emission du 5 avril 1984 de Jean Montalbetti, sur France-Culture: Entretien avec Nathalie Sarraute, cité in Jacques Lecarme \& Eliane Lecarme-Tabone (1997: 15).

3 Ibid.

4 Entretien avec Serge Fauchereaux et Jean Ristat, "Conversation avec Nathalie Sarraute", Digraphe, 1984, 32, p. 10, cité par Monique Gosselin (1996: 25-26). 
tance pré-linguistique capable de traduire "ces quelques moments, quelques mouvements [...] encore intacts, assez forts pour se dégager de cette couche protectrice qui les conserve" (Sarraute, 1983: 227). Même besoin de se justifier chez Alain Robbe-Grillet qui, après avoir longtemps condamné la littérature du "moi", avoue dans Le Miroir qui revient son désir d'explorer une nouvelle voie:

Le moment est venu d'avancer sur d'autres pistes, et de retourner comme un gant la belle théorie nouvellement promue, afin de débusquer la bureaucratie renaissante qu'elle nourrit en cachette [...] Il devient urgent de tout remettre en cause, et [...] de s'interroger à nouveau sur le rôle ambigu que jouent, dans le récit moderne, la représentation d'une personne qui est à la fois un corps, une projection intentionnelle et un inconscient (Robbe-Grillet, 1984: 12).

Un objectif qu'il atteint en préservant malgré tout une certaine marge d'ambiguïté sur la nature de son projet puisqu'après avoir déclaré dès les premières pages: “c'est encore dans une fiction que je me hasarde ici", il se contredit un peu plus loin en admettant éprouver "un certain plaisir à utiliser la forme traditionnelle de l'autobiographie" (Robbe-Grillet, 1984: 13 et 16). Si Robbe-Grillet avoue ainsi son "projet de raconter [s]a vie", il parle en même temps d"“essai critique" et de "roman" (Robbe-Grillet, 1984: 18). En dépit de leur originalité et modernité, Enfance de Nathalie Sarraute et Le Miroir qui revient d'Alain Robbe-Grillet se conforment donc aux diverses conventions du genre autobiographique sans pour autant cesser de se protéger contre l'accusation d"“autobiographisme"s.

Publié également dans les années 80 et résistant à toute classification, L'Amant (1984) de Marguerite Duras se situerait d'après le tableau dressé par Lejeune dans Le pacte autobiographique sur les combinaisons possibles des récits autodiégétiques, dans la case "indéterminé" où "non seulement le personnage n'a pas de nom, mais l'auteur ne conclut aucun pacte, - ni autobiographique, ni romanesque" (Lejeune, 1975: 28-29). Si d'un côté Duras annonce que “c'est la première fois qu'[elle] n'écri[t] pas une fiction", de l'autre elle soutient: "L'histoire de votre vie, de ma vie, elle n'existe pas ou bien alors il s'agit de lexicologie. Le roman de ma vie, de nos vies, oui, mais pas l'histoire" (Armel, 1990a: 28-29). Dans ce cas, pourquoi ne pas avoir accompagné le titre L'Amant par la mention "roman"? “On m'a demandé de mettre 'roman"”, explique Duras, “j'ai dit que je pouvais le mettre et puis je ne l'ai pas mis" (Armel, 1990b: 18). Les raisons qu'elle donne, par conséquent, pour justifier son hésitation n'ont rien à voir avec un choix autobiographique. Préférant "la sécheresse du blanc" au sous-titre "roman", l'auteur laisse à son lecteur la liberté de décider: "qu'on dise 'roman' ou non", déclare-t-elle, “au fond, ça les regarde, les lecteurs" (Armel, 1990b: 18). C'est surtout, comme le constate Franciska Skutta, parce qu'“on peut s'appuyer sur des indications extérieures à l'œuvre, à savoir des données biographiques contenues dans des ouvrages critiques et des interviews avec l'auteur" que l'on peut considérer L'Amant une

5 Néologisme employé par Aliette Armel (1990b: 32). 
autobiographie (Skutta, 1987: 79). Mais ce n'est sûrement pas Duras qui confirmera ce choix, préférant l'indétermination à la classification.

Plus récente, l'œuvre d'Annie Ernaux reste difficile à définir. Si l'auteur refuse d'employer le mot "fiction" pour définir son écriture -"fiction" étant un mot qui, d'après elle, offre la possibilité de "se protéger", de se masquer en disant “j'ai tout inventé" (Ernaux, 2003: 27)-, ce n'est pas non plus ouvertement le mot "autobiographie" qu'elle emploie pour décrire ses récits: "autobiographie... J'hésite beaucoup", avoue-t-elle dans L'écriture comme un couteau:

C'est l'écriture, globalement, qui détermine le degré de vérité et de réalité pas seulement l'emploi du 'je' fictionnel ou autobiographique. Il y a pas mal de récits autobiographiques qui donnent une insupportable impression de manquer la vérité. Et des textes dits romans qui l'atteignent (Ernaux, 2003: 30).

Ernaux se défend aussi d'identifier son écriture à ce genre hybride qu'est l'autofiction. Dans Le vrai lieu, elle explique en effet: "je me suis toujours révoltée contre l'assimilation de ma démarche d'écriture à l'autofiction parce que dans le terme même il y a quelque chose de replié sur soi, de fermé au monde" (Ernaux, 2014: 106). Aussi préfère-t-elle parler dans le cas de Les Années d'“autobiographie impersonnelle" et éliminer son "je" pour laisser place à un "elle" (Ernaux, 2014: 75). En somme, un désir de retranscrire un temps ressenti, le reflet d'un monde à travers son expérience personnelle, une écriture de la mémoire et de la réalité, au plus proche d'une vérité sociologique ou ethnologique qui amène d'ailleurs l'auteur à employer les néologismes "auto-socio-biographie" ou encore "auto-ethnologique" (Ernaux, 2003: 23). Finalement, le point commun entre tous ces récits est moins l'appartenance à un genre, l'autobiographie, que la volonté d'éluder l'appartenance à un genre particulier.

\section{Contre le genre autobiographique}

Non seulement la plupart des autobiographies étudiées dans cet article cherchent-elles à résister à toute définition, mais elles brouillent volontairement les frontières entre autobiographie, biographie, journal et roman, une distance prise aussi bien avec le genre qu'avec le "je". C'est à chaque fois une nouvelle façon d'écrire son "moi"que Claude Roy invente dans ses trois volumes autobiographiques Moi je (1970), Nous (1972) et Somme toute (1976). Sous-titrés "Essai d'Autobiographie", l'éditeur explique en effet sur la couverture du dernier volume que Claude Roy "utilise[nt] tous les genres pour exprimer toutes les facettes de la vie": "chronique d'un témoin, confession, journal, analyses, documents, portraits, poèmes en prose (et en vers), réflexions et maximes" sont en effet les composantes de cette trilogie (1976).

Brouiller les frontières entre autobiographie, autoportrait, biographie et fiction est 
ce qu'entreprend habilement Roland Barthes dans son Roland Barthes par Roland Barthes (1975), un titre paradoxal qui suggère déjà la complexité du "je"/“jeu" où "Roland Barthes", auteur critique, se distancie de "Roland Barthes", sujet biographique. Car il s'agit non seulement d'une distance prise avec le genre de la collection, la biographie, mais aussi avec le genre qu'il suggère en plaçant deux fois son nom, l'autobiographie. Un "Roland Barthes puissance deux", où l'auteur, à la fois sujet, objet et modèle de son récit ajoute une autre complication en annonçant dès l'ouverture: "Tout ceci doit être considéré comme dit par un personnage de roman". Une épigraphe qui déconcerte dès l'entrée tout espoir de voir Barthes se livrer et qui lui permet de révoquer toute question de fidélité par rapport à tout ce qu'il s'apprête à raconter. Barthes se déguise ainsi en personnage fictif et se permet de déclarer:

C'est un roman, mais pas une biographie [...] Je me suis mis en scène comme un personnage de roman, mais qui n'aurait pas de nom propre, en quelque sorte, et à qui il n'arriverait pas d'aventure à proprement parler romanesque (Brochier, 1975: 32).

En somme, Barthes se met en scène comme "une troisième personne qui ne renvoie cependant à aucune créature fictive", et Roland Barthes par Roland Barthes se présente comme un essai qui "s'avoue presque un roman : un roman sans noms propres" (Barthes, 1975: 124). Mais le "presque" ou le "comme si" de l'épigraphe concernent "uniquement l'énonciation": 1'“énoncé", affirme Lejeune, "continue à être soumis aux règles strictes et propres du contrat autobiographique" (Lejeune, 1980: 34). Autrement dit, l'épigraphe ne signifie pas que le sujet du discours est une personne fictive, mais que le narrateur se pose "comme" un personnage fictif. Supposons alors, comme le propose Tzvetan Todorov, que "plutôt que le romancier authentique d'une histoire fictive, Barthes [serait] l'énonciateur inauthentique d'histoires (ou de discours) vraies" (Todorov, 1981: 324). Non seulement l'auteur flirte avec le roman, mais la structure du Roland Barthes par Roland Barthes composée de fragments trouble aussi efficacement les frontières entre autobiographie et autoportrait. Difficile alors pour le lecteur, après tous ces degrés d'écriture, d'atteindre ce "degré zéro" de lecture où il découvrirait l'auteur dans toute sa vérité. Une formule qui peut toutefois aider à définir ce récit est celle que propose Lejeune qui l'interprète comme "un retour à la situation autobiographique dans le cadre d'une collection 'biographique' qui elle-même prétendait a l'origine reconstituer l'autoportrait de l'auteur' (Lejeune, 1980: 50).

Même désir de mêler et autobiographie, biographie, journal et roman chez Yves Navarre. En choisissant d'intituler son récit Biographie (1981), de la sous-titrer "roman", d'annoncer dès les premières pages "ma vie, comme toute vie, est un roman", et de la présenter comme le roman de tous ses romans antérieurs, comme le récit de leur genèse puis commencer à rédiger les premières pages sous la forme d'un journal, Navarre pose dès l'entrée la nature hétéroclite de son projet. À la fois journal du récit qu'il écrit et écriture d'une biographie de sa vie, Biographie mêle autobiographie, biographie et journal: tout un assemblage qui 
produit, d'après l'auteur, un "roman", mais un "roman au plus proche de [s]a vie" (Navarre, 1981: 13). Il s'agit en effet d'une narration complexe qui alterne première et troisième personne du singulier, mais où le protagoniste porte le même nom que le narrateur et l'auteur, identité qui caractérise indubitablement l'autobiographie.

C'est paradoxalement un désir d'authenticité qui pousse Alain Bosquet à sous-titrer L'enfant que tu étais (1982) un "roman". Conscient de la part de partialité qu'implique tout récit de son passé, l'auteur préfère admettre qu'il "réinvente" son "petit ami": "pour le moins, nous en serons ensemble étonnés: lui, de se découvrir un peu autre; moi, de croire que l'imagination remplace les faits perdus" (Bosquet, 1982: 10). Comment, avoue en effet Bosquet, ne pas “éviter l'attendrissement, ni les pièges de la mémoire, ni la rage qui vient aux hommes quand ils tentent en vain de redevenir des enfants"? Difficile. L'enfant que tu étais serait ainsi, comme le suggère l'introduction, un récit qui donnerait une "dimension romanesque à l'histoire vraie d'un petit garçon" (Bosquet, 1982: 4).

Quant à Marguerite Duras, difficile de savoir dans L'Amant (1984) où commence l'autobiographie et où finit la fiction. En déclarant dès les premières pages: "L'histoire de ma vie n'existe pas. Ça n'existe pas. Il n'y a pas de centre. Pas de chemin, pas de ligne" (Duras, 1984: 14), Duras se place en effet dans la lignée de ces autres autobiographes "masqués" comme Yves Navarre et Roland Barthes, qui exposent dès l'entrée le statut ambigu de l'entreprise qu'ils s'apprêtent à réaliser. Semblable à Navarre, qui assigne à sa Biographie le sous-titre "roman", ou à Barthes, qui nie pareillement le projet autobiographique de son Roland Barthes par Roland Barthes en le définissant "un roman" (Brochier, 1975: 32), Duras affirme que L'Amant, loin d'être 1' "histoire de [s]a vie", est "le roman de [s]a vie" (Armel, 1990a: 28). C'est bien une "nouvelle approche" qu'envisage Duras lorsqu'elle affirme vouloir parler “de certains enfouissements qu' [elle] aurait opéré sur certains faits" (Duras, 1984: 14). Ayant parlé jusqu'alors des "périodes claires, de celles qui étaient éclairées", l'auteur souhaite à présent révéler les "périodes cachées de cette même jeunesse" (Duras, 1984: 14). Un effort, remarque Franciska Skutta, “qu'il n'est pas possible d'exiger de la seule mémoire, de sorte que, pour la narratrice, le seul moyen de révéler les secrets du passé ou de recréer un fait dont le souvenir s'est éteint sera de recourir à l'imagination, à l'écriture" (Skutta, 1987: 86). Semblable au "Moi par moi" de Barthes, L'Amant serait donc aussi le «programme de l'imaginaire". Mais si pour Barthes l'imaginaire est une "façon quasi romanesque de se vivre comme un personnage intellectuel, dans la fiction, dans l'illusion et nullement dans la vérité" (Brochier, 1975: 32), pour Duras il serait plutôt question de vivre à la frontière entre "vérité" et "fiction", ou plutôt, comme dirait Michel Foucault dans son Histoire de la sexualité, "faire fonctionner des fictions à l'intérieur de la vérité” (in Armel, 1990b: 23). La vérité que l'on atteint alors dans ces récits est donc une vérité toute personnelle où, comme dirait Grisi:

Les oppositions réel/imaginaire, réalité/fiction ou reproduction/invention ne paraissent plus pertinentes pour distinguer littérature romanesque et littérature autobiographique 
[...] L'écriture autobiographique n'a donc plus à être évaluée sur un critère d'exactitude ou de vérité historique, vérifiable et idéalement objective [...] A l'extérieur et au vérifiable se mêle tout ce qui est de l'ordre de la réalité interne, psychique, subjective. (Grisi, 1996: 55).

Difficile alors de savoir si Duras est, comme dirait Marcelle Marini, "passée aux aveux"; ce que l'on sait c'est qu'elle est "entrée dans la littérature de l'aveu" où le "moi" constitue le fondement de son œuvre et l'imaginaire son outil de création littéraire (Marini, 1985: 8).

Journal, roman et rêve sont aussi les ingrédients de cette autre autobiographie qu'est Mes Parents (1986) d'Hervé Guibert, où l'association curieuse des mots "personnage" et "autobiographie" choisis par l'éditeur en quatrième de couverture pour définir le récit, ne fait que renforcer l'ambiguïté sur la nature du livre que le lecteur s'apprête à entamer: "Hervé Guibert devient un personnage de son livre, le temps d'une autobiographie de jeunesse" (1986). Même confusion dans la déclaration faite par Hervé Guibert au sujet de A l'ami qui ne m'a pas sauvé la vie (1990): "Même celui qui est Guibert dans le livre est un personnage",; ou encore dans le texte placé au dos de la couverture de Le Protocole Compassionnel signé "H.G. ", et qui signale qu'on y "retrouve les mêmes personnages: Hervé Guibert, écrivain malade du sida, ses proches, la communauté des malades et de leurs soignants" (1991). Ambiguïté surtout dans le sous-titre "roman" choisi par l'auteur pour définir ces deux derniers récits et qui trouble, comme dans les cas précédents, les frontières et limites de l'autobiographie.

C'est également le sous-titre "roman" que Christophe Bourdin choisit pour définir son récit Le Fil (1994) et c'est aussi, comme Yves Navarre, une partie de son journal qu'il insère dans une des trois parties qui composent son autobiographie. Or Le Fil n'est ni "roman", ni journal, mais un texte hybride qui mêle journal, rêve et autobiographie et raconte en trois "temps": "temps des hypocondries", de "l'agonie" et du "rêve", les stades qui ont ponctué la maladie de ce jeune auteur atteint du sida. Que l'auteur ait choisi de sous-titrer son récit "roman", au lieu d'autobiographie, serait à relier à la composition particulière du récit, qui non seulement mêle les genres mais emploie respectivement trois pronoms personnels pour illustrer son rapport avec la maladie. Difficile ici de parler de récit de vie, lorsque la maladie, l'agonie et la mort sont les thèmes qui composent cet écrit. Si la mort est, de surcroît, ce qui attend l'auteur à la fin du récit, plus qu'une autobiographie, il s'agirait de ce que Jean-Pierre Boulé appelle une "thanatographie" (Boulé, 1999: 207) ou, comme dirait plus subtilement Stéphane Grisi, une «autopathographie", un concept qui "emprunte la forme littéraire de l'autobiographie et le fond thématique de la pathologie" et qu'il définit comme: "tout écrit autobiographique dans lequel l'auteur évoque de façon centrale ou périphérique, des faits,

6 Entretien avec A. de Gaudemar, "La vie du sida", Libération, 1 mars 1990, p. 19, cité in Jean-Pierre Boulé (1999: 193). 
des idées ou des sentiments relatifs à sa propre maladie" (Grisi, 1996: 25). Ce que le soustitre "roman" pourrait donc aussi insinuer serait une tentative d'adoucir une réalité, de rendre moins dure la triste lecture de cet homme irrémédiablement condamné. En décourageant le lecteur à faire le lien entre le récit et sa vie, Bourdin évite d'en dramatiser leur fin, inévitablement unie. Semblable à la fin onirique du récit, la définition "roman" autoriserait l'évasion, le détachement par le rêve et l'imagination.

Confusions et contradictions semblent être le mode d'emploi de la plupart des autobiographies mentionnées ici pour éviter tout risque d'être classées. Placées en effet au début de leurs récits, ces précautions constituent une première ligne de défense de l'auteur contre l'emprise interprétative du lecteur. Pour d'autres, l'imprécision ou le doute autour du genre en question suggèrent au contraire un désir d'authenticité, la difficulté de trouver le terme juste capable de saisir leur vérité.

\section{Contre le "je" autobiographique}

Si les autobiographies examinées dans cet article hésitent ou évitent tout simplement de s'identifier à ce genre qui les définit, elles rajoutent une autre entrave à cette définition, en accompagnant ou substituant leur "je” par d'autres pronoms. Or, l'identité de l'auteur, du narrateur et du personnage que requiert l'autobiographie, se marque le plus souvent par l'emploi de la première personne du singulier. C'est ce que Gérard Genette (1972) appelle la narration "autodiégétique" dans sa classification des "voix" du récit qu'il établit à partir des œuvres de fiction. Le "je"représente, en effet, celui qui tient la plume pour raconter son histoire, et celui qu'il était à l'époque des faits narrés. Le lecteur perçoit ainsi un mouvement de va-et-vient entre les deux “je”, identifiables par l'emploi des temps verbaux auxquels ils sont respectivement liés: présent pour le narrateur et passé pour le personnage. Quoi de plus naturel pour l'autobiographe que l'emploi du “je" pour exprimer son histoire, sa vie. C'est bien l'avis que partage Michel Leiris dans cette véritable apologie du "pronom JE", inclue dans sa première œuvre autobiographique Aurora:

Il m'est toujours plus pénible qu'à quiconque de m'exprimer autrement que par le pronom JE: non qu'il faille voir là quelque signe particulier de mon orgueil, mais parce que ce mot JE résume pour moi la structure du monde. Ce n'est qu'en fonction de moimême et parce que je daigne accorder quelque attention à leur existence que les choses sont. (Leiris, 1946: 39-40).

Sans égaler forcément Leiris dans ce délire d"“orgueil", innombrables sont les auteurs qui n'envisagent ou ne conçoivent s'exprimer autrement que par le pronom personnel "je". Or, l'emploi du "je" n'est pas une condition suffisante pour certifier la nature autobiographique du texte. Le récit peut être écrit à la première personne, sans que le narrateur soit confondu avec le personnage principal. Il s'agit alors de ce que Genette nomme la narration 
"homodiégétique" et qui s'applique, d'après Lejeune, au "récit de témoin", ou à la "biographie à la première personne" (Lejeune, 1975: 18). Mais un autre cas peut aussi se présenter: celui où le récit est écrit à la première personne, sans que l'auteur soit confondu avec le narrateur. Il s'agit là de ce que l'on appelle le roman à la première personne ou roman personnel. Si, comme le constate justement Grisi, “le 'je' de la narration n'est pas une condition suffisante de la littérature autobiographique, il n'en représente pas davantage une condition nécessaire" (Grisi, 1996: 46). A ce sujet Lejeune rajoute qu' "il suffit de continuer ce raisonnement pour voir qu'en sens inverse il peut parfaitement y avoir identité du narrateur et du personnage principal sans que la première personne soit employée” (Lejeune, 1975: 16). Rien n'empêche, en effet, certains autobiographes à employer la deuxième ou la troisième personne du singulier pour désigner leur "je” présent ou passé.

Paul Valéry n'écrivait-il pas: "Le moi se dit moi ou toi ou $i l$. Il y a les trois personnes en moi. La Trinité. Celle qui tutoie le moi ; celle qui le traite de Lui" (Valéry, 1973: 440). Aussi, à condition que l'identité de l'auteur, narrateur et personnage principal soit respectée, il peut très bien exister des autobiographies écrites à la deuxième, troisième personne du singulier ou du pluriel. "Cette identité”, précise encore Lejeune, “n'étant plus établie à l'intérieur du texte par l'emploi du 'je', est établie indirectement, mais sans aucune ambiguïté, par la double équation: auteur = narrateur, et auteur = personnage, d'où l'on déduit que narrateur = personnage même si le narrateur reste implicite" (Lejeune, 1980: 16). La substitution partielle ou totale du pronom personnel "je" par un autre pronom personnel du singulier ou du pluriel ne signifie donc pas que le genre du récit a changé. Il s'agit toujours d'une autobiographie: le personnage dont parle le récit est toujours l'auteur du texte, et le pacte entre auteur et lecteur est plus ou moins respecté. C'est le cas des autobiographies étudiées dans cette quatrième et dernière partie, qui emploient le "tu", le "il", mais parfois aussi le "nous" ou le "vous" comme substitution partielle ou totale de la première personne du singulier.

\subsection{Emploi exclusif, alterné ou exceptionnel de la deuxième personne du singulier}

Rares sont les autobiographies entièrement écrites dans un autre pronom que le "je": il s'agit le plus souvent d'un emploi exceptionnel ou d'une alternance de pronoms dans des autobiographies qui utilisent aussi la première personne du singulier. Certains auteurs s'y sont néanmoins risqués, comme Alain Bosquet qui, dans L'Enfant que tu étais (1982), parvient habilement à employer d'un bout à l'autre de son autobiographie la deuxième personne du singulier pour "parler" à l'enfant qu'il a été. Annoncé dans le titre même du récit, le tutoiement est une manière pour l'auteur de "montrer" à son "doux compagnon" son "affection" (Bosquet, 1982: 10). Une "évocation au vocatif” qui permet d'autre part à Bosquet de donner une certaine autonomie à ce "moi” passé et respecter la distance qui sépare le petit garçon de l'adulte. Comment, suggère justement l'auteur, employer la même personne pour parler 
d'un enfant qu'il n'est plus? Impossible de "parler de lui à la première personne" du singulier explique-t-il: “je ne m’arroge pas le droit d'affirmer que je suis encore, à mon âge, ce qu'il [l'enfant] était autrefois" (Bosquet, 1982: 10). Pas plus à la première qu'à la troisième personne du singulier, car, précise-t-il, “j’aurais de la peine à le traiter à la troisième: il n'est pas un étranger avec qui je veuille tenir d'inutiles distances" (Bosquet, 1982: 10). La deuxième personne du singulier est donc employée ici pour renforcer une distance temporelle et affective.

C'est un dialogue fictif entre les deux voix de son "moi" que met en scène Nathalie Sarraute dans Enfance (1983), où le "tu” et le "je" représentent deux instances présentes, deux voix intérieures qui cherchent, à travers le dialogue, à restituer certains "moments" ou "mouvements" du passé et un langage capable de les évoquer. Un dédoublement intérieur où le “je” n'est donc plus quelqu'un qui possède la parole comme une faculté innée que l'auteur se contente d'exercer, mais quelqu'un à qui le "tu" donne la parole pour raconter quelque chose qu'elle n'a encore jamais exprimé. À travers le dialogue, c'est donc aussi une recherche que met en scène Sarraute, un travail de construction où le "tu", semblable au "meneur de jeu" dans la recherche philosophique par discussion, conduit son interlocuteur "je" à découvrir le savoir qu'elle porte en elle:

- Et c'est tout? Tu n'as rien senti d'autre? [...]

- C'est vrai... je dérangeais leur jeu.

- Allons, fais un effort...

- Je venais m'immiscer... m'insérer là où il n'y avait pour moi aucune place.

- C'est bien, continue...

- J'étais un corps étranger... qui gênait...

- Oui: un corps étranger. Tu ne pouvais pas mieux dire (Sarraute, 1983: 75).

Dans son récit autobiographique Le Fil (1994), ce sont successivement trois pronoms personnels que Christophe Bourdin emploie pour se désigner. Le récit évoque un passé récent de Bourdin: le moment qui a précédé et suivi la déclaration de cette maladie. Il s'agit donc d'une période assez brève, étendue sur une dizaine d'années, où l'auteur reflète sur les répercussions que le sida a eu sur sa vie. Dans la première partie de son récit intitulée le "temps des hypocondries", où l'auteur fait la découverte de sa séropositivité, c'est le "tu" qu'il utilise pour narrer son "je” passé. Mais c'est dans un tout autre contexte et pour des motifs différents à ceux de Sarraute que l'auteur a recours ici à la deuxième personne du singulier. Si pour l'auteur de Enfance il s'agit de créer un dialogue fictif entre deux instances d'un "moi" présent qui se penche sur son passé, pour le jeune écrivain de Le Fil, l'emploi exclusif du "tu" lui permet de suggérer l'impossible identité entre son "je" présent et malade et un “je” passé encore ignorant sa séropositivité. Aucun dialogue, donc, dans Le Fil, mais une narration à la deuxième personne du singulier où le "je" implicite du narrateur s'adresse à son “je" passé pour établir un bilan, un constat. Le constat d'un temps révolu, d'une santé 
perdue, de deux modes de vie opposés, une fois la maladie déclarée et assumée. Plus qu'une distance temporelle, ce que Bourdin suggère à travers le "tu" serait plutôt un dédoublement intérieur entre une partie de son "moi” resté sain, et l'autre souffrant:

Il semblait que tu fusses composé de deux individus incompatibles, fractionné en deux hommes successifs, juxtaposés dans le temps [...] qui s'excluaient mutuellement, aux émotions contradictoires impossibles à éprouver simultanément (Bourdin, 1994: 58-59).

L'emploi du “tu” serait donc justifié par la difficulté de l'auteur d'affirmer qu'il est encore celui qu'il était autrefois, soit un sujet apparemment intouchable, alors qu'il est se sent désormais un sujet malade. C'est la première personne du singulier qui domine en revanche la deuxième partie, intitulée le "temps de l'agonie", où Bourdin recopie une partie de son journal et note au jour le jour sa coexistence avec la maladie. Suit une dernière partie, "le temps du rêve", où l'auteur s'unit à un être imaginé pour vivre à la deuxième personne du pluriel et au conditionnel, des instants de liberté rêvée. Confiées à un temps irréel, les dernières pages de Le Fil emportent en effet le "vous" de l'auteur et d'un personnage imaginé dans un univers fantasmé.

\subsection{Emploi exclusif, alterné ou exceptionnel de la troisième personne du singulier}

L'autobiographie rédigée entièrement à la troisième personne du singulier existe aussi. Comme le signale encore Grisi, c'est le cas du livre de Louis Wolfson intitulé Le Schizo et les langues (1970), où l'emploi du «pronom 'il', pour se désigner, crée une atmosphère impersonnelle, et rend palpable le clivage entre le sujet parlant et le sujet en action» (Grisi, 1996: 47). C'est la première personne du pluriel que choisit, en revanche, Maxime Montel pour écrire son récit autobiographique sur le sida Un Mal imaginaire (1994). Par l'emploi exclusif de ce "nous", explique Grisi, "l'auteur désigne la communauté de destin et de souffrance des malades atteints de l'infection fatale", le sida (Grisi, 1996: 158). De même que pour la deuxième personne du singulier, ces emplois exclusifs du "ill" et du "nous" sont assez exceptionnels, plus courants sont les emplois alternés ou occasionnels des pronoms personnels singuliers ou pluriels.

C'est pour exposer l'impossible unité et l'intolérable division de son “moi” qu'André Gorz a recours au "il" dans Le Traître (1958). L'alternance de première et troisième personnes du singulier d'un bout à l'autre de son autobiographie lui permet en effet d'exposer son sentiment d'illégitimité et d'insécurité dans la rassurante unité que suggère la première personne du singulier. L'intérêt de cette narration à double pronom est de montrer le progrès d'un "moi" en construction: en partant du constat de son "moi” fracturé et aliéné, l'auteur cherche à reconstruire son identité. Le but est évidemment la victoire du "je" sur le "il", le moment où l'auteur n'a plus besoin d'être escorté par son double de papier pour parler à la 
première personne du singulier. Le "il" est donc dans Le Traître une instance contemporaine au "je” qui s'alterne avec elle jusqu'au moment où l'auteur est capable d'assumer son statut de sujet.

Le recours à la troisième personne du singulier dans les deux derniers volumes autobiographiques de Claude Roy Nous (1972) et Somme toute (1976) est en revanche exceptionnel. Dans son deuxième volume Nous, l'auteur emploie le "ill" et son prénom "Claude" pour se distancer de son "je" passé. La fonction de la troisième personne du singulier servirait en effet à renforcer une distance temporelle et à souligner le regard paternaliste que Claude Roy jette sur son passé, où "qui je ne suis plus observe qui je suis avec un détachement enjoué, bienveillant" (Roy, 1970: 68). C'est, au contraire, pour suggérer la division de son "je” présent que l'auteur emploie la troisième personne du singulier pour ouvrir et clore son troisième volume Somme Toute. Ici, un face-à-face amusant entre l'auteur et son double sert à expliciter la division de son "je" présent et l'impossible expression de son identité à travers cette seule et rassurante entité que représente "la pauvre première personne du singulier" (Roy, 1976: 16). Ayant cherché, comme il l'explique dans son premier volume Moi je, "à savoir qui je fus, qui je suis, et quel moi est ce je" (Roy, 1970: 234), Claude Roy découvre à la fin de sa trilogie que le "Il" est le pronom qui définit son "dernier moi": "Il disait autrefois: 'Moi je'. Il voulut dire: 'Nous'. Il est maintenant tenté de dire, réduit à dire Il“" (Roy, 1976: 443).

Quant à Roland Barthes, non seulement évite-t-il de définir le genre de son Roland Barthes par Roland Barthes (1975), mais il essaie aussi, comme suggère Lejeune, "d'échapper à la pesanteur du moi par des variations incessantes sur les pronoms personnels" (Lejeune, 1980: 50). En construisant un jeu où il est tantôt désigné par un "je", tantôt par un "ill" et parfois même un "nous" et un "vous", l'auteur fait de tout pour maintenir une aura d'ambiguïté autour de sa vraie identité. Ce que lui permettent en effet tous ces jeux de pronoms est d'empêcher son “je” de trop s'exposer. Avec Roland Barthes par Roland Barthes, le lecteur se situe donc dans ce que Lejeune appelle, au sujet de l'œuvre de Gide, la "complication", soit “le jeu complice où l'ambiguïté de l'énonciation se greffe sur la complexité de l'énoncé" (Lejeune, 1975: 167). L'ambiguïté implique le double sens d'un mot ou d'une expression: elle se situe au niveau de l'énonciation et reflète l'incertitude où se trouve le lecteur de la position du narrateur par rapport à ce qu'il raconte. En multipliant les jeux de l'énonciation et de l'identité ce n'est plus comme pour André Gorz ou Claude Roy la construction du sujet que cherche ici Barthes, mais sa déconstruction, en rendant visible la "fissure du sujet" (Barthes, 1975: 5).

À la fois "je" dans les chapitres de son journal et "il" dans les chapitres de sa biographie, Yves Navarre se sert en revanche de la troisième personne pour séparer son "je" présent de son "je" passé. La fonction du "il" dans Biographie (1981) est donc de suggérer une distance temporelle, mais aussi, comme l'indique le titre, d'imiter le genre biographique, une séparation qu'il poursuit également dans le genre adopté pour narrer ces deux "je": le 
journal pour le premier, et la biographie pour le deuxième, où l'auteur devient "Yves". Si le "il" désigne le personnage biographique, soit Yves Navarre à l'époque des faits narrés, ce pronom se réfère aussi parfois au narrateur biographique, à cette instance fictive que l'auteur a créée pour narrer ces chapitres de biographie. Ce qui distingue alors le personnage du narrateur est le recours au prénom "Yves" pour le premier et l'allusion au "biographe" pour le second. Conformes au sens premier du mot "autobiographie", ces chapitres sont la biographie de l'auteur écrite par lui-même. Un glissement qui s'effectue d'autant plus "facilement", constate Lejeune, les deux genres ayant "une large zone de recouvrement, le texte autobiographique étant souvent déjà lui-même une traduction 'à la première personne' du texte biographique conventionnel" (Lejeune, 1980: 42).

Désignant aussi bien son "je" passé que son "je” présent, la troisième personne du singulier employée par Marguerite Duras dans L’Amant (1984) est plus complexe. Non seulement suggère-t-elle la distance temporelle mais elle semble aussi illustrer cette ligne presque invisible qui sépare pour l'auteur la réalité de la fiction. Ce que cherche en effet Duras n'est pas, comme pour Roland Barthes, à déconstruire son "moi”, mais plutôt retrouver une image de soi passée et créer à travers l'écriture, comme André Gorz et Yves Navarre, une nouvelle identité. Mais loin de se soucier à préserver, comme Nathalie Sarraute, le souvenir intact, sans intrusion du présent, ce que désire Duras est de récupérer le souvenir de son histoire d'amour avec l'amant, en rapportant tous les sentiments que ce souvenir provoque sur son “je” présent. S'il s'agit bien d'une récupération d'un moment passé, c'est une récupération qui n'hésite pas à avoir recours à l'invention pour restituer non pas l'image exacte et objective du passé, mais une évocation subjective du passé, l'image que l'auteur conserve à présent de ce même instant. En choisissant de narrer à la troisième personne du singulier toute la fin de L'Amant, y compris le dernier paragraphe qui se réfère à un passé récent, Duras anticipe la technique adoptée plus tard dans L'Amant de la Chine du Nord où auteur, narrateur et personnage sont tous désignés par le pronom personnel "elle". Un "elle" qui permet encore une fois de brouiller les frontières entre autobiographie et fiction, et qui permet à Duras de déclarer ironiquement: "Je suis redevenue un écrivain de romans" (Duras, 1991: 12).

La deuxième ou troisième personne du singulier peut aussi suggérer non plus une distance intérieure entre les deux instances d'un même "moi", mais une distance "extérieure"entre le "je" de l'auteur et cet "autre" que le miroir, le regard d'autrui ou la photographie lui renvoient. Le "il" employé parfois par Hervé Guibert dans A l'ami qui ne m'a pas sauvé la vie (1990) ou Le Protocole Compassionnel (1991) servirait à expliciter cette distance et décrire son "moi" confronté à cet "autre" que lui renvoient le miroir ou le regard d'autrui et suggérer en même temps son incrédulité face à son corps altéré:

Je ne peux pas dire non plus que j'avais de la pitié pour ce type, ça dépend des jours, parfois j'ai l'impression qu'il va s'en sortir [...] d'autres fois il est clair qu'il est condamné, en route vers la tombe, inéluctablement (Guibert, 1991: 18-19). 
Même chose pour les photographies: ce que voit Guibert dans les photographies de lui prises par Hans Georg Berger est "toute la distance accomplie dans le passage d'un je à un $i l$, et bien d'autres distances" (Guibert, 1992). Une distance qu'explicite aussi Barthes dans son Roland Barthes par Roland Barthes en employant le "nous", "vous" ou "il" pour désigner son "moi" photographié. Le "il" servirait donc à exprimer le contraste entre image intérieure et image extérieure, en d'autres mots, l'image d'un corps qui ne correspond pas à l'idée que l'auteur se fait de lui.

C'est l'emploi exclusif de la troisième personne du singulier que choisit Annie Ernaux dans son récit autobiographique Les Années (2008) pour suggérer son désir d'inscrire dans l'Histoire des années 50 à l'an 2000 l'existence d'une femme et mêler sa mémoire individuelle à la mémoire collective. En commentant une photo d'elle en février 92 et remémorant son désir de "réunir ces multiples images d'elle, séparées, désaccordées, par le fil d'un récit, celui de son existence", l'auteur avoue que le choix entre l'emploi "je" ou le "elle" devient son souci principal:

Il y a dans le "je" trop de permanence, quelque chose de rétréci et d'étouffant, dans le “elle" trop d'extériorité, d'éloignement. L'image qu'elle a de son livre, tel qu'il n'existe pas encore, l'impression qu'il devrait laisser, est celle [d'] une coulée de lumière et d'ombre sur des visages (Ernaux, 2008: 187-188).

Dans les dernières pages du livre, là où le sujet de l'énoncé se fond presque avec le sujet de l'énonciation, où le "elle" passé est sur le point de devenir le "je” présent de l'auteur, Ernaux conclut: "Aucun "je" dans ce qu'elle voit comme une sorte d'autobiographie impersonnelle - mais "on" et "nous" - comme si, à son tour, elle faisait le récit des jours d'avant" (Ernaux, 2008: 252). L'emploi exclusif de la troisième personne du singulier permet donc à l'auteur de marquer l'écart temporel entre les différentes instances de son "moi" et suggérer surtout son désir de "rendre la dimension vécue de l'Histoire" (Ernaux, 2008: 251). C'est encore le "elle" que Ernaux emploie dans son dernier récit autobiographique Mémoire de fille (2016), une troisième personne du singulier que l'auteur alterne avec la première pour souligner, comme dans Les Années, une distance temporelle, mais surtout sa difficulté à s'identifier à cette fille de 58 et son désir de s'en détacher:

J'ai voulu l'oublier aussi cette fille. L'oublier vraiment, c'est-à-dire ne plus avoir envie d'écrire sur elle. Ne plus penser que je dois écrire sur elle, son désir, sa folie, son idiotie et son orgueil, sa faim et son sang tari (Ernaux, 2016: 17).

Comment, se demande plus loin Ernaux, "fondre la fille de 58 et la femme de 2014 en un 'je'? ”. Impossible. Il s'agira donc pour l'auteur de "dissocier la première de la seconde par l'emploi de "elle" et de "je", pour aller le plus loin possible dans l'exposition des faits et des actes" (Ernaux, 2016: 23). En employant la troisième personne du singulier, conclut 
l'auteur: "Je ne construis pas un personnage de fiction. Je déconstruis la fille que j'ai été" (Ernaux, $2016: 60)$.

Finalement, à de plus ou moins faibles degrés, tous les pronoms personnels du singulier ou du pluriel peuvent être employés dans l'autobiographie. Du moment que l'identité de l'auteur, narrateur et personnage est respectée, rien n'empêche l'autobiographe de désigner son "je” présent ou passé par un autre pronom que la première personne du singulier. L'intérêt? Expliciter une distance, mettre au premier rang l'impossible unité, l'inévitable division du sujet. Lorsque l'autobiographe choisit, en effet, d'accompagner ou substituer son "je"par "tu" ou "il", son désir est d'expliciter un écart qui peut servir à suggérer: 1) une distance temporelle entre le "je" présent de l'auteur et son "je" passé; 2) une distance intérieure ou dédoublement entre deux instances d'un même "moi"; 3) une distance extérieure entre le "je" de l'auteur et cet "autre" que lui renvoient le miroir, la photographie ou le regard d'autrui. Qu'elle soit temporelle, intérieure ou extérieure, la distance que suggère l'emploi de la deuxième ou troisième personne du singulier aboutit en général à deux résultats: à la construction ou déconstruction d'une image de soi. Une image qui finalement est toujours le fruit d'une construction savamment élaborée, la création d'une image de soi qui est celle que l'auteur voudrait que le lecteur ait de son "moi".

\section{Conclusion}

Philippe Lejeune constate, au sujet des Dialogues, Rousseau juge de Jean-Jacques, que le "procès" reconstitué fictivement par Jean-Jacques Rousseau où l'auteur fait dialoguer "les rôles de l'accusation et de la défense", ne se propose pas de "construire un point de vue sur soi”, mais d'en “détruire un” (Lejeune, 1980: 55). Il s'agirait en effet, d'après Lejeune, d'un montage bien construit, qui fournirait à Rousseau l'occasion de se reconstruire une nouvelle image, en détruisant celle qui le tournait en désavantage. Evidemment, "les choses tournent bien sûr à l'avantage de l'autobiographe qui fait triompher peu à peu sa véritable image" (Lejeune, 1980: 55). Ce que tous ces exemples de récits autobiographiques français de ces dernières 50 années démontrent, que ce soit à travers le soupçon, la subversion ou la transformation du genre, est leur désir de nouveauté, de trouver une écriture capable de refléter leur véritable image. Un souci de vérité dont le résultat ne sera toujours qu'à demi sincère non seulement, comme le suggère André Gide, parce que “tout est toujours plus compliqué qu'on ne le dit" (Gide, 1926: 280), ou comme dirait Annie Ernaux, "la vérité est simplement le nom donné à ce qu'on cherche et qui se dérobe sans cesse” (Ernaux, 2003: 30), mais surtout, comme affirme Roland Barthes, parce que "qui parle (dans le récit) n'est pas qui écrit (dans la vie) et qui écrit n'est pas qui est” (Barthes, 1966: 20). "Qui est” restera toujours la question, aussi bien pour l'auteur que pour le lecteur de ces "jeux" de pronoms. 
VERS UNE NOUVELLE AUTOBIOGRAPHIE: SUBVERSIONS ET TRANSFORMATIONS DU GENRE DANS...

\section{Referencias bibliográficas}

Armel, Aliette. 1990a. "Le jeu autobiographique" in Magazine Littéraire, n 278, 28-31.

Armel, Aliette. 1990b. Marguerite Duras et l'autobiographie. Pantin, Le Castor Astral.

BARTHES, Roland. 1966. "Introduction à l'analyse structurale des récits" in Communication, $\mathrm{n}^{\mathrm{o}} 8,1-27$.

Barthes, Roland. 1975. Roland Barthes par Roland Barthes. Paris, Editions du Seuil coll. "Ecrivains de Toujours".

Beaujour, Michel. 1980. Miroirs d'encre: Rhétorique de l'autoportrait. Paris, Editions du Seuil, coll. "Poétique".

Bosquet, Alain. 1982. L'Enfant que tu étais. Paris, Editions Grasset \& Fasquelle.

BoulÉ, Jean-Pierre. 1999. Hervé Guibert: Voices of the Self. Liverpool, Liverpool University Press.

Bourdin, Christophe. 1994. Le Fil. Paris, Gallimard.

Brochier, Jean-Jacques. 1975. "Vingt mots-clés pour Roland Barthes" in Magazine Littéraire, $\mathrm{n}^{\circ}$ 97, 28-37.

DARrieussecQ, Marie. 1997. “De l'autobiographie à l'autofiction, Mes Parents, roman ? « in Ralph Sarkonak (textes réunis et édités par), Le corps textuel de Hervé Guibert, La Revue des Lettres Modernes. Paris-Caen, Minard.

Doubrovsky, Serge. 1977. Fils. Paris, Galilée.

Duras, Marguerite. 1984. L'Amant. Paris, Editions de Minuit.

Duras, Marguerite. 1991. L'Amant de la Chine du Nord. Paris, Gallimard.

ErnaUX, Annie. 2003. L'écriture comme un couteau. Entretien avec Frédéric-Yves Jeannet. Paris, Gallimard.

Ernaux, Annie. 2008. Les Années. Paris, Gallimard.

Ernaux, Annie. 2014. Le vrai lieu. Entretiens avec Michelle Porte. Paris, Gallimard.

Ernaux, Annie. 2016. Mémoire de fille. Paris, Gallimard.

Forrester, Vivianne. 1983. «Portrait de Nathalie» in Magazine Littéraire, nº 196, 18-24.

Genette, Gérard. 1972. Figures III. Paris, Editions du Seuil.

GIDE, André. 1926. Si le grain ne meurt. Paris, Gallimard.

Gorz, André. 1958. Le Traître. Paris, Editions du Seuil. 
Gosselin, Monique. 1996. Monique Gosselin présente Enfance de Nathalie Sarraute. Paris, Gallimard.

Green, Julien. 1963. Partir avant le jour. Paris, Bernard Grasset.

Grisi, Stéphane. 1996. Dans l'intimité des maladies: de Montaigne à Hervé Guibert. Paris, Desclée de Brouwer.

Guibert, Hervé. 1981. L'Image fantôme. Paris, Editions de Minuit.

Guibert, Hervé. 1984. Le Seul visage. Paris, Editions de Minuit.

Guibert, Hervé. 1986. Mes Parents. Paris, Gallimard.

Guibert, Hervé. 1990. A l'ami qui ne m’a pas sauvé la vie. Paris, Gallimard.

Guibert, Hervé. 1991. Le Protocole Compassionnel. Paris, Gallimard.

GuiBert, Hervé. 1992. “L'Image de soi, ou l'injonction de son beau moment”, préface de Dialogues d'images, Hans Georg Berger. Bordeaux : William Blake.

JACCOMARD, Hélène. 1993. Lecteur et lecture dans l'autobiographie française contemporaine: Violette Leduc, Françoise d'Eaubonne, Serge Doubrovsky, Marguerite Yourcenar. Genève, Droz.

Lecarme, Jacques \& Lecarme-Tabone, Eliane. 1997. L'Autobiographie. Paris, Armand Colin/Masson.

LeIRIS, Michel. 1939. L'Age d'homme. Paris, Gallimard.

LeIrIs, Michel. 1946. Aurora. Paris, Gallimard.

LeIrIs, Michel. 1966. Fibrilles. Paris, Gallimard.

LeJeune, Philippe. 1975. Le pacte autobiographique. Paris, Editions du Seuil, coll. "Poétique".

LeJeune, Philippe. 1980. "L'autobiographie à la troisième personne", Je est un autre: l'autobiographie de la littérature aux médias. Paris, Editions du Seuil, coll. "Poétique".

MARINI, Marcelle. 1985. “Une femme sans aveu” in $L$ 'Arc, $\mathrm{n}^{\circ} 98$.

Montel, Maxime. 1994. Un Mal imaginaire. Paris, Editions de Minuit, coll. "Au vif du sujet”.

Navarre, Yves. 1981. Biographie. Paris, Editions Flammarion.

Robbe-Grillet, Alain. 1984. Le Miroir qui revient. Paris, Editions de Minuit.

Roy, Claude. 1970. Moi je. Paris, Gallimard.

Roy, Claude. 1972. Nous. Paris, Gallimard.

Roy, Claude. 1976. Somme Toute. Paris, Gallimard. 
Anales de Filología Francesa, n. ${ }^{\circ}$ 27, 2019

VERS UNE NOUVELLE AUTOBIOGRAPHIE: SUBVERSIONS ET TRANSFORMATIONS DU GENRE DANS...

Sarraute, Nathalie. 1983. Enfance. Paris, Gallimard.

SkutTA, Franciska. 1987. "L'Amant de Marguerite Duras: une autobiographie?" in Cahiers $d u \operatorname{Cerf} X X, \mathrm{n}^{\mathrm{o}} 3,77-90$.

Todorov, Tzvetan. 1981. "Le dernier Barthes” in Poétique, n 47, 323-327.

VALÉRY, Paul. 1973. Cahiers. Tome I. Paris, Gallimard.

Wolfson, Louis. 1970. Le Schizo et les langues. Paris, Gallimard, coll. "Connaissance de l'Inconscient'". 